\title{
AVALIAÇÃO MULTICRITERIAL NO MAPEAMENTO DA SUSCETIBILIDADE DE DESLIZAMENTOS DE TERRA ${ }^{1}$
}

\author{
Hilton Luís Ferraz da Silveira² ${ }^{2}$ Carlos Alberto Vettorazzi ${ }^{3}$ e Roberta Averna Valente ${ }^{4}$
}

\begin{abstract}
RESUMO - No mapeamento da suscetibilidade de deslizamentos de terra, necessita-se agregar as características da paisagem que atuam de maneira distinta no processo de queda de barreira e podem representar especificidades de uma região. Nesse contexto, o objetivo deste trabalho foi avaliar o método da Média Ponderada Ordenada (MPO) para o mapeamento de áreas suscetíveis ao deslizamento de terra. O método pressupõe a integração de diferentes características da paisagem (fatores), com a associação de pesos a elas (pesos de fator - $p f$ ). Pf representa a importância desses fatores para o estudo. Utilizaram-se, neste estudo, os fatores: substrato geológico, solos, declividade do terreno, uso e cobertura do solo e precipitação. Na definição dos fatores e seus pesos, empregou-se a Técnica Participatória. Para avaliação da MPO, geraram-se dois mapas de suscetibilidade, calculando o risco de tomada de decisão (R) e nível de compensação entre fatores (C). Para o primeiro, consideraram-se apenas pf e a compensação total entre fatores $(C=100 \%)$ e para o outro, um segundo grupo de pesos (de ordenação (po), que caracteriza a influência dos fatores). Os valores de pó resultaram em valor de C $=72 \%$. No primeiro mapa predomina o nível de suscetibilidade médio, por influência excessiva do fator declividade (fator mais influente; com o maior de $p f$ ). No segundo, como consequência de po houve a agregação dos fatores de acordo com a importância previamente definida para eles e a melhor distribuição, em relação ao primeiro, de área entre os níveis de suscetibilidade. Concluiu-se que a MPO é adequada ao mapeamento da suscetibilidade de deslizamento, e a coerência entre $p f$ e po confere robustez ao método.
\end{abstract}

Palavras-chave: Suporte à decisão; Combinação Linear Ponderada; Média Ponderada Ordenada.

\section{MULTICRITERIA EVALUATION IN THE MAPPING OF LANDSLIDE SUSCEPTIBILITY}

\begin{abstract}
The mapping of landslides susceptibility requires the aggregation of landscape characteristics that act differently in the process of landslides and may represents specificities of a region. In this context, the general objective of this study was to evaluate the Ordered Weighted Averaging (OWA) method in the mapping of landslide susceptibility. The method presupposes the integration of different characteristics of the landscape (factors), associating weights to them (named Importance Weights - IW). IW express the importance of these factors to the study. In the study, we used the factors: geological substrate, soil type, slope, landuse and land-cover, and precipitation. In the definition of factors and their weights, the Participatory Technique was used. In order to evaluate the OWA, two susceptibility maps were generated, calculating the decision making risk ( $R$ ), and compensation level among factors (C). For the first one, only the IW and the total compensation among factors were considered $(C=100 \%)$ and for the other one, a second group of weights (the Order Weights (OW), that represents the influence of factors). Using the OW we obtain $C=72 \%$. In the first map the medium level of susceptibility was predominant, by excessive influence of the slope factor (the most influent factor; with the highest IW). In the second map, by influence of $O W$, there was the aggregation of factors according to the importance predefined for them and the best distribution, in relation to the first one, of area between susceptibility levels. We conclude that OWA is adequate to mapping the landslide susceptibility and that the coherence between IW and OW is responsible to determine the robustness to the method.
\end{abstract}

Keywords: Decision support; Weighted Linear Combination; Ordered Weighted Averaging.

\footnotetext{
${ }^{1}$ Recebido em 14.03.2014 aceito para publicação em 02.10.2014.

${ }^{2}$ Empresa Brasileira de Pesquisa Agropecuária - EMBRAPA, CNPS, UEP Recife. E-mail: <alquires@hotmail.com>.

${ }^{3}$ Departamento de Engenharia de Biossistemas, Escola Superior de Agricultura "Luiz de Queiroz" - ESALQ/USP. E-mail: <cavettor@usp.br>.

${ }^{4}$ Departamento de Ciências Ambientais, Universidade Federal de São Carlos - UFSCAR. E-mail: <roavalen@ufscar.br. >.
} 


\section{INTRODUÇÃO}

Os deslizamentos de terra são responsáveis pela morte e soterramento de pessoas todos os anos, em especial no período chuvoso. Associado ao risco de morte, há o problema ambiental, que é gerado pela remoção do solo, e o favorecimento da erosão. Dessa forma, frequentemente se têm associados aos deslizamentos os prejuízos econômicos e ambientais, os danos materiais e a manutenção de alto custo, bem como lesões ou fatalidades (DAS et al., 2010). Esses danos podem ser atenuados se as relações de causa e efeito dos eventos forem conhecidas (INTARAWICHIAN; DASANANDA, 2010). O mapeamento da suscetibilidade de deslizamentos de terra está entre as soluções para compreender e prever perigos futuros, a fim de mitigar as suas consequências. Segundo Lei e Jing-feng (2006), esse tipo de estudo retrata a distribuição espacial futura de uma encosta desmoronar. Cervi et al. (2010) afirmaram que o processo de mapeamento de suscetibilidade pode ser baseado em métodos estatísticos ou determinísticos, o qual envolve a identificação de regiões suscetíveis à ruptura do talude, tendo por base as propriedades geológicas e geomorfológicas da região avaliada.

Os fatores que influenciam nesse processo, no entanto, são inúmeros. Os mais comumente mencionados são o tipo de solos, a declividade, a precipitação, o uso e cobertura do solo e o substrato geológico, entre outros (TOMINAGA et al., 2005; YALCIN, 2008; DAS et al., 2011; FEIZIZADEH et al., 2013). O método dito indireto permite integrar esses fatores no mapa de suscetibilidade e, ainda, inclui a importância desses fatores no processo, com base na experiência de especialistas no assunto (LEI; JING-FENG, 2006; MALCZEWSKI, 2006). Nesse contexto, podem-se citar os métodos de Avaliação Multicriterial (AM), os quais são considerados um avanço ao procedimento convencional de cruzamento de planos de informação, justamente por sua capacidade de integrar conjuntamente diferentes fatores (VALENTE; VETTORAZZI, 2011). O método da Média Ponderada Ordenada (MPO) representa a evolução conceitual da AM, por ser flexível e possibilitar a obtenção de soluções, para o processo decisório, com diferentes níveis de risco para a tomada de decisão Boroushaki e Malczewski (2010). Nas soluções avessas a risco (um local deve atender a todos os critérios para ser incluído no conjunto de decisão) e naquelas totalmente arriscadas (um local será incluído no conjunto de decisão se pelo menos um critério for atendido) (BOROUSHAKI; MALCZEWSKI, 2010).

Chan (2011) mapeou as áreas prioritárias ao desenvolvimento da agricultura por meio da MPO. Gorsevski et al. (2012) pesquisaram a melhor alocação de aterros sanitários na região de Polog, na Macedônia. Meng et al. (2011) e Feizizadeh e Blaschke(2013) utilizaram a MPO para avaliar áreas suscetíveis a deslizamento, respectivamente, no Canadá e no Irã.

Nesse contexto, o objetivo geral do trabalho foi avaliar o método da Média Ponderada Ordenada para o mapeamento de áreas suscetíveis ao deslizamento de terra. O objetivo específico foi avaliar a importância e influência dos fatores selecionados para esse processo decisório.

\section{MATERIAL E MÉTODOS}

\section{1. Área de estudo}

O Município de Juquiá localiza-se no Vale do Ribeira, na região de governo de Registro, na porção Sul do Estado de São Paulo, entre os paralelos $24^{\circ} 01^{\prime} 42^{\prime \prime S}$

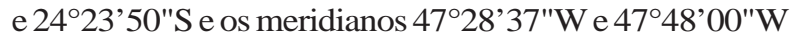
(Figura 1), ocupando uma área de aproximadamente $865 \mathrm{~km}^{2}$. A vegetação original da região (floresta nativa) ocupa 77\% do município, sendo o restante de sua área ocupada em 17,5\% por vegetação rasteira, 1,5\% por áreas agrícolas e 0,5\% por áreas antrópicas e 3,5\% por corpos d'água (SILVEIRA et al., 2008), como ilustrado na Figura 1. É uma região prioritária para estudos de suscetibilidade de deslizamentos, porque congrega condições ambientais que influem nos processos de movimentação de massas em taludes. Fatores que agravam essa condição são a falta de recursos para a prevenção do desmatamento e a ocupação urbana desordenada.

Predominam no município as rochas précambrianas, entre elas os migmatitos, granitos, quartzitos e mármores dolomíticos, do complexo gnáisico-migmatítico e do Grupo Açungui, com maior participação dos metassedimentos do tipo filitos, ardósias, calcários, calcários epimetamórficos, quartzitos diversos e mármores dolomíticos (LEPSCH et al., 1999). Quanto à geomorfologia, é dividido nas províncias Escarpa do Planalto e Serraria Costeira (LEPSCH et al., 1999). A Escapa do Planalto caracteriza-se por extrema fragilidade 


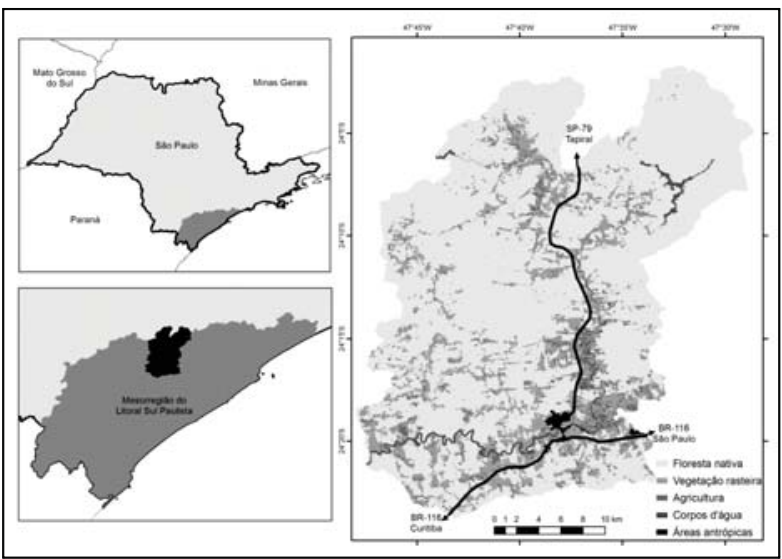

Figura 1 - Uso e cobertura do solo e localização do Município de Juquiá, SP.

Figure 1-Land-use/land-cover and location of the municipality of Juquiá, SP.

erosiva, em virtude das altas declividades e do avançado estágio de intemperismo das rochas, estando sujeito a constantes ocorrências de movimentos de massa. Já a Serraria Costeira é formada por espigões e morros isolados ou ligados à escarpa do planalto, sendo marcante a quebra do relevo. Isso como resultante das características litólicas, tectônicas e erosivas dessa província (LEPSCH et al., 1999)

O solo tipo Cambissolo está relacionado aos substratos geológicos, principalmente os de rochas magmáticas e metamórficas e predomina na porção central ao Norte do município. Está presente, ainda, em pequena porção do Sudeste do município, onde é limitado por uma faixa longitudinal de Gleissolo (LEPSCH et al., 1999). Na porção Sul, ele ocorre na forma de manchas isoladas, visto que da porção central a essa direção há a presença de Podzólicos, de solo Orgânico nas planícies de inundação de alguns rios e de solo Aluvial, em especial, nos fundos dos vales de outros rios do município.

O clima da região é, segundo a classificação de Köppen, do tipo Cfa, caracterizado como tropical úmido, em que não há a ocorrência de estação seca além de uma média térmica para o mês mais quente sempre acima dos $22^{\circ} \mathrm{C}$ (LEPSCH et al., 1999).

\subsection{Avaliação Multicriterial (AMC)}

Para o mapeamento de risco de deslizamentos com a abordagem multicriterial, empregou-se o método da
Média Ponderada Ordenada (MPO), no ambiente de um SIG (IDRISI Kilimanjaro). O método teve sua conceituação matemática estabelecida por Yager (1988) e envolve a aplicação de dois grupos de pesos; os de fatores e os de ordenação, além da definição do próprio conjunto de fatores. O primeiro relaciona-se à importância do fator no processo de tomada de decisão, e o segundo controla a maneira pela qual os fatores são agregados e controlam, ainda, o nível de compensação entre os fatores e o risco assumido no processo de tomada decisão (MALCZEWSKI, 1999).

\subsection{Critérios}

Na definição dos critérios (fatores e restrições) e, posteriormente, dos pesos de fatores, empregouse a Técnica Participatória (EASTMAN, 2003), por meio da consulta de 11 especialistas das áreas de conservação de ecossistemas, de geoprocessamento, geologia e de climatologia, além de uma revisão bibliográfica. Os fatores considerados importantes aos objetivos do trabalho foram:

(1) Substrato geológico: gerado a partir da Carta Geológica do Brasil ao Milionésimo (Folha Iguape SG 23), produzida pelo Serviço Geológico do Brasil (SCHOBBENHAUS et al., 2004). Os substratos geológicos foram classificados de acordo com o nível de propensão à formação de cicatrizes de escorregamento, que se constitui em um plano homogêneo sem qualquer resistência à movimentação do solo, como observaram Fernandes et al. (2004). O aspecto geológico de uma região caracteriza a intemperização e, dessa forma, o grau de estabilidade dos elementos sólidos que constituem a cadeia (SOARES, 2005). Com base nos trabalhos de Alheiros et al. (2003), Ayalew et al. (2005) e Soares (2005), definiram-se, para esse processo decisório, os grupos de rochas sedimentares, metamórficas e magmáticas e seus pesos, respectivamente, baixo, médio e alto. Isso considerando a influência de cada tipo de rocha na formação das cicatrizes de escorregamento. Em seguida, normalizou-se o mapa geológico para a escala de 0-255 bytes, usando função linear crescente. Assim, quanto menores os valores (mais próximos a 0), menor o risco associado ao fator.

(2) Solos: os solos arenosos possuem risco alto de erosão, porém um risco baixo de deslizamento de terra, enquanto os solos com textura mais argilosa apresentam comportamento contrário (BERTONI;

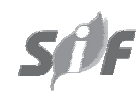

Revista Árvore, Viçosa-MG, v.38, n.6, p.973-982, 2014 
LOMBARDI NETO, 2008). Para esse critério, os solos (LEPSCH et al., 1999) foram previamente hierarquizados, considerando sua textura, uma vez que essa característica contempla não só o tamanho do grão, como Alheiros et al. (2003) ressaltaram, mas também as proporções entre os grãos no solo. As cinco classes geradas foram reclassificadas para a escala de 0 -255 bytes, por meio de uma função linear crescente (Tabela 1).

(3) Declividade do terreno: é um dos fatores mais importantes na ocorrência dos deslizamentos de terra, sendo um dos fatores mais empregados em estudos sobre deslizamentos de terra (MANTOVANI et al., 2000; SMYTH; ROYLE, 2000; ALHEIROS et al., 2003; USGS, 2003; REMONDO etal., 2005; FEIZIZADEH; BLASCHKE, 2012). Para a produção desse fator, utilizaram-se os dados do projeto Brasil em Relevo produzido pela Embrapa Monitoramento por Satélite (MIRANDA, 2005), os quais foram padronizados para a resolução espacial de 20 $\mathrm{m}$, por meio de re-amostragem e método do Vizinho Mais Próximo. Os valores de altimetria foram convertidos em valores de declividade e, posteriormente, normalizados para a escala de 0-255 bytes, por meio de uma função linear crescente.

(4) Uso e cobertura do solo: a importância da vegetação para manutenção da segurança das encostas é explicada por Catani et al. (2005), que afirmaram que a vegetação exerce papel fundamental na estabilização das encostas, uma vez que, como salientou Carpi Júnior (2001), o sistema radicular preenche os vazios do solo, retardando o processo de infiltração das águas das chuvas. Para esse autor, o suporte mecânico radicular faz que a resistência do solo aumente, proporcionalmente, com a densidade de raízes. Ao contrário, nas áreas de cultivo a baixa cobertura foliar e o sistema radicular pequeno nos primeiros 30 dias do desenvolvimento das culturas coincidem com os meses de chuvas intensas, principalmente no Sul do Brasil, ocorrendo, assim, uma fase crítica nesse período, no qual normalmente se verificam $80 \%$ das perdas de solos dos ciclos anuais (CARPI JUNIOR, 2001). Para esse critério, o mapa de uso e cobertura do solo, produzido por Silveira et al. (2008), foi atualizado em campo (exatidão global de 95\%) e reclassificado de acordo com a importância relativa das suas classes nos processos de deslizamento de terra (RAUTELA; LAKHERA, 2000; CATANI et al., 2005), como pode ser observado na Tabela 2 . Em seguida, os níveis de risco foram normalizados, por meio de uma função linear crescente, para a escala de 0-255 bytes.

(5) Precipitação: destaca-se o seu fundamental papel nos processos de deslizamentos de terra, uma vez que o solo saturado perde sua coesão natural (ALHEIROS et al., 2003). Os dados das estações pluviométricas presentes na área de estudo foram importados e transformados em pontos cotados, utilizando as médias dos meses de maior precipitação, que são dezembro, janeiro, fevereiro e março (valores que correspondiam melhor ao fenômeno estudado). Assim, produziu-se um mapa de distribuição da precipitação (interpolação pelo método da triangulação inscrita em uma circunferência) e, a partir dele, o mapa de fator associado ao risco que a chuva traz na saturação do solo. Este último, na escada de 0-255 bytes, sendo os maiores valores de precipitação associados aos maiores riscos de deslizamento, por meio de função linear crescente.

\subsection{Pesos de fator (pf)}

Os pesos de fator expressam a importância relativa de cada fator, no grupo de fatores em consideração (EASTMAN, 2003; MALCZEWSKI, 1999). Nessa etapa foi, portanto, inserida ao processo decisório a preferência dos especialistas (Técnica Participatória), em relação à ordem de importância dos fatores. Obteve-se a seguinte ordem de importância dos fatores: declividade do terreno, uso e cobertura do solo, substrato geológico,

Tabela 1 - Classes de suscetibilidade em função da textura dos solos, no Município de Juquiá, SP.

Table 1 - Classes of susceptibility according to soil texture, in the municipality of Juquiá, SP.

\begin{tabular}{|c|c|c|}
\hline Solo & Textura em relação à argila e areia & Suscetibilidade \\
\hline Podzólico Vermelho Amarelo Álico & argilosa a muito argilosa & muito alta \\
\hline Cambissolos Álicos & argilosa a muito argilosa (misturas de cascalhos) & alta \\
\hline Cambissolos distróficos & argilosa ou média argilosa & média-alta \\
\hline Gleissolos & argilosa média/argilosa com substrato arenosos. & média-baixa \\
\hline Solos Aluviais & argilosa média e arenosa & baixa \\
\hline Solos Orgânicos & Indiscriminada: com depósitos aluviais arenosos. & muito baixa \\
\hline
\end{tabular}

Revista Árvore, Viçosa-MG, v.38, n.6, p.973-982, 2014 
Tabela 2 - Suscetibilidade a deslizamentos de terra para os tipos de uso e coberturas do solo do Município de Juquiá, SP. Table 2 - Landslide susceptibility to the land-use/land-cover, in the municipality of Juquiá, SP.

\begin{tabular}{lc}
\hline Uso e cobertura do solo & Suscetibilidade \\
\hline Cobertura florestal: mata nativa e vegetação de brejo & baixa \\
Vegetação rasteira: pastagens e campos & média-baixa \\
Agricultura: banana, palmito juçara e plantas ornamentais & média-alta \\
Áreas antrópicas: cidades, casas, rodovias & alta \\
\hline
\end{tabular}

precipitação e solos. Considerando essa ordem de importância e baseando-se no método da Comparação Pareada (CP), desenvolvido por Saaty (1980) no contexto do Processo Hierárquico Analítico (AHP), calcularam-se os pf. O método CP baseia-se na elaboração de uma matriz de comparação entre os fatores, de acordo com a importância relativa entre pares de fatores (EASTMAN, 2003). Para a matriz (Tabela 3), os fatores são comparados, dois a dois, utilizando como referência uma escala contí-nua de nove pontos e classificados de acordo com a importância relativa entre eles, previamente estabelecida por meio da Técnica Participatória. O valor 1 indica que dois fatores são “igualmente" importantes, enquanto o valor 9 aponta que um fator é “extremamente” mais importante do que os demais.

A consistência da matriz é avaliada por sua Taxa de Consistência (TC), que, segundo Saaty (1980), indica a probabilidade de os valores de comparação entre os fatores ser gerada aleatoriamente. Segundo esse autor, os valores de TC devem estar sempre abaixo de 0,10 . Nesse contexto, os valores adequados de $\boldsymbol{p} \boldsymbol{f}$, para a suscetibilidade a deslizamentos, foram definidos considerando a ordem de importância dos fatores (Técnica Participatória) e, ainda, de modo a obter valor de TC inferior a 0,10 (Tabela 3).

\subsection{Pesos de ordenação (po)}

Geraram-se mapas de suscetibilidade de deslizamento de terra para dois cenários, calculando os respectivos riscos de decisão $(\mathrm{R})$ e nível de compensação entre fatores (C), como proposto por Valente e Vettorazzi (2008). O risco é conceituado por Eastman (2003) como a probabilidade de que a decisão tomada esteja errada, resultando das diversas incertezas a que essa tomada de decisão pode estar sujeita, como erros na base de dados e na regra de decisão. A compensação pode ser pensada como taxa de substituição, ou seja, quanto de um fator o tomador de decisão se dispõe a abrir mão, em favor de uma "quantidade" especificada de algum outro fato assumido (VALENTE; VETTORAZZI, 2013).

Para o primeiro cenário, associou-se igual po $(p o=0,2)$ para os fatores. De acordo com Boroushaki e Malczewski (2008), nesse cenário tem-se uma atitude neutra, visto que $\mathrm{R}=0,5$ e, por consequência, ocorre a compensação total entre os fatores. Essa situação representa a alternativa (cenário) correspondente ao método tradicional de AMC, denominado Combinação Linear Ponderada (CLP). A neutralidade da situação indica que não houve compensação entre os fatores e, assim, prevalecem os pf. Isso significa que os fatores são agregados considerando apenas a sua importância

Tabela 3 - Matriz de comparação pareada do mapeamento de áreas suscetíveis a deslizamentos de terra, no Município de Juquiá, SP.

Table 3 - Pairwise comparison matrix used in the mapping of areas that are susceptible to landslides, in the municipality of Juquiá, SP.

\begin{tabular}{|c|c|c|c|c|c|c|}
\hline & F1 & F2 & F3 & F4 & F5 & Pesos de fator (pf) \\
\hline F1 & 1 & & & & & 0,0618 \\
\hline F2 & 2 & 1 & & & & 0,0973 \\
\hline F3 & 3 & 2 & 1 & & & 0,1599 \\
\hline F4 & 4 & 3 & 2 & 1 & & 0,2625 \\
\hline F5 & 5 & 4 & 3 & 2 & 1 & 0,4185 \\
\hline
\end{tabular}

Taxa de Consistência (TC) $=0,0231,0000$

Sendo: F1 = solos; F2 = precipitação; F3 = substrato geológico; F4 = uso e cobertura do solo; e F5 = declividade do terreno. Where: $F 1=$ soil; F2 = precipitation; F3 = geological substrate $; \mathrm{F} 4=$ Land-use/land-cover; and F5 = slope. 
para o processo decisório. Com o intuito de ter um conjunto de pesos de ordenação, proporcionando um cenário distinto de CLP, mas mantendo a importância dos fatores para o processo de tomada de decisão, gerou-se o segundo cenário (mapa de suscetibilidade) considerando os seguintes pesos de ordenação para os fatores: 0,125 para o uso e cobertura do solo; 0,250 para a precipitação; 0,300 para o substrato geológico; 0,025 para a declividade; e 0,300 para o tipo de solo. Esses pesos foram definidos considerando os fatores, segundo sua ordem de influência nesse processo decisório: declividade do terreno, uso e cobertura do solo, substrato geológico, precipitação e solos.

\section{RESULTADOS}

Para o primeiro mapeamento (cenário) de suscetibilidade a deslizamento, o qual corresponde agregação dos fatores por meio do método tradicional da CLP ( $\mathrm{R}=0,5 ; \mathrm{C}=100 \%)$, obteve-se o Município de Juquiá, com 44,5\% de sua área associada ao nível de suscetibilidade médio e, como ilustrado na Figura 2a, espacialmente distribuído por seus limites. Os níveis de suscetibilidade muito baixo, baixo, alto e muito alto ocupavam, respectivamente, 7,3\%, 26,3\%, 4,32\% e 17,57\% da área do município. Os maiores níveis de suscetibilidade representaram apenas $22 \%$ da área de Juquiá.

No segundo mapeamento houve compensação (C) de $72 \%$ entre os fatores e, mesmo assim, continuouse com risco médio $(\mathrm{R}=0,5)$ para a tomada de decisão (Figura 2b). De acordo com esse mapa, o município apresenta distribuição de áreas suscetíveis com nível variando de muito baixo a muito alto de, respectivamente, 7,2\%; 18,4\%; 37,5\%; 22\%; e 14,9\%. As classes de suscetibilidade baixa, média e alta foram aquelas com maiores alterações de valores, e maiores níveis de suscetibilidade passaram a ocupar aproximadamente 37\% do município.

\section{DISCUSSÃO}

No primeiro cenário (Figura 2a), as classes com suscetibilidade muito alta e alta distribuem-se, predominantemente, do Norte do município à sua porção média. Nesse mapa, tem-se a influência marcante do fator declividade. Isso em razão de o fator ser o mais influente no processo decisório e, ainda, do peso de fator $(0,4185)$ associado a ele. Os demais fatores tiveram

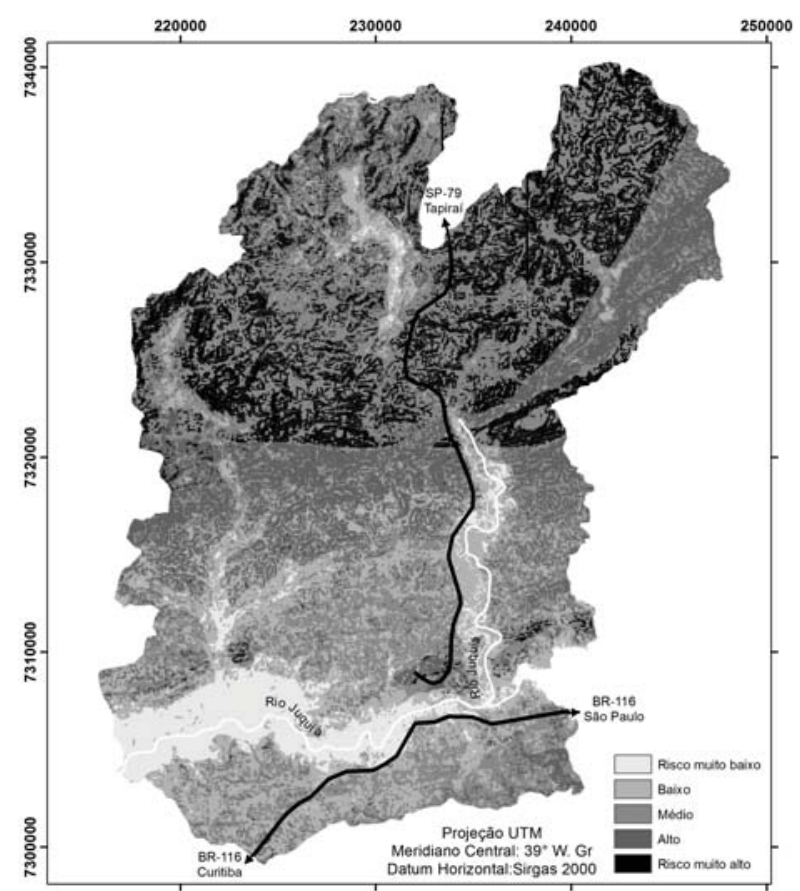

Figura 2 - Áreas suscetíveis a deslizamentos de terra, no Município de Juquiá, SP, obtidas por: (a) CLP ( $\mathrm{r}$ $=0,5 ; \mathrm{c}=100 \%)$; e (b) MPO ( $\mathrm{r}=0,5 ; \mathrm{c}=72 \%)$.

Figure 2-Areas that are susceptible to landslide, in the municipality of Juquiá, SP, produced by (a) WLC $(r=0.5 ; C=$ 100\%) and; (b) OWA ( $r=0,5 ; C=72 \%)$.

sua influência neutralizada, mesmo sendo importantes para o mapeamento. O único fator que conseguiu manter sua influência foi o substrato geológico (terceira posição no ranqueamento de influência). Sua presença pode ser notada na porção Nordeste do município, onde ocorre uma zona de contato entre substratos diferentes, marcados por uma longa falha (Figura 2). Boroushaki e Malczewski (2008) também observaram, em mapa com risco médio (método CLP), a excessiva influência de fatores com maior ranqueamento.

O mapa com risco médio (compensação total entre os fatores) caracteriza-se por apresentar soluções nem arriscadas nem avessas a risco, conforme citado por Malczewski (2000) e Valente e Vettorazzi (2008). Nem sempre suas soluções são as ideais porque, como pode ser observado na Figura 2a, sofre muito influência do fator de maior ranqueamento. Fernandes et al. (2001) ressaltaram que, embora muito importante, a declividade não deve ser o único fator a ser considerado nos estudos referentes a deslizamentos de terra. Esses autores citaram 
que, em alguns trabalhos em que o fator declividade foi o único critério considerado, o mapeamento acabou por considerar terrenos de menor declividade como de baixa suscetibilidade, não condizendo com a realidade, uma vez que trabalhos de campo mostram com frequência a presença de deslizamentos, principalmente, na forma de corridas de massa.

No segundo mapeamento (Figura 2b) houve alteração na distribuição das classes de suscetibilidade, em especial daquelas associadas aos níveis de suscetibilidade muito alto e alto. Elas tiveram sua distribuição mais bem definida, com a distinção entre elas. Mesmo ocupando a porção Norte a porção média do município se tem a predominância da classe alta nas regiões Noroeste e Central do município. Essa nova distribuição reflete os pesos de ordenação associados aos fatores e, portanto, a maneira como foram compensados (pesos de ordenação e pesos de fator). Os menores pesos de ordenação foram associados aos fatores de maior influência no processo decisório e, ainda, respeitando a hierarquização entre eles e a importância que têm para o objetivo do trabalho. Por esse motivo, o fator declividade, considerado o mais importante (maior peso de fator $=0,4185$ - Tabela 3) e também o de maior influência no processo, foi aquele que recebeu o menor valor de peso de ordenação $(0,025)$.

O segundo menor valor de peso de ordenação $(0,100)$ foi atribuído ao fator classificado como segundo em ordem de importância (0,2625 - Tabela 3) e influência. Em contrapartida, os fatores com menor influência no processo foram associados aos maiores valores de peso de ordenação, mais uma vez respeito-se a hierarquia entre eles. Pode-se assim dizer que houve, para a produção desse mapa de risco médio, alta compensação entre os pesos de fator e ordenação. Mesmo reduzindo a influência do fator declividade, notou-se sua contribuição no mapa final, em especial quando se observou a distribuição dos níveis de suscetibilidade por meio de uma reta no sentido norte-sul do município. $\mathrm{Na}$ porção Nordeste do município, tem-se uma predominância do nível de maior suscetibilidade, justamente como consequência de essa porção estar associada aos maiores percentuais de declividade.

A mesma condição se tem para o fator uso e cobertura do solo, com presença marcante na caracterização dos níveis de suscetibilidade média e baixa (associado a baixas declividades), como nas planícies e nas vertentes dos vales dos rios Juquiá (Figuras 1 e 2a) e afluentes, uma vez que é nesses locais onde se concentram os processos de desmatamento, plantio e criação de gado. Nas planícies do rio Juquiá existiam áreas de Mata Atlântica preservadas, as quais foram associadas à classe muita baixa, enquanto no trecho a montante (que está às margens da Rodovia SP-79 - Figuras 1 e 2a) predominava a suscetibilidade baixa. Essas áreas representam aproximadamente $26 \%$ do total do município e, mesmo sob os menores níveis de suscetibilidade, merecem atenção em razão da marcante presença de desenvolvimento urbano e rural (áreas antrópicas Figura 1), justamente por consequência de suas próprias características (relacionadas aos critérios).

No que diz respeito à precipitação, há uma faixa de maior precipitação de sua porção Oeste a Nordeste, a qual foi associada à suscetibilidade alta e, ou, muito alta. Já na região com menor precipitação (com média mensal nos meses considerados em torno de 230 a 250 mm de chuva) houve redução nos níveis de suscetibilidade.

Nesse contexto, o mapeamento (cenário 2 - Figura 2b) possibilitou, a partir da integração dos diferentes critérios, a espacialização dos níveis de deslizamento de acordo com a realidade do município, sendo, para isso, necessária a ponderação da influência dos critérios. Dessa forma, o nível de suscetibilidade a deslizamentos passou a representar aquele proposto (pelos especialistas) para os diferentes critérios.

\section{CONCLUSÕES}

A partir dos resultados, sobre a área de estudo foi possível concluir que:

1. AAvaliação Multicriterial com o método da Média Ponderada Ordenada é adequada ao mapeamento da suscetibilidade de deslizamento de terra. A utilização do método é, contudo, fortemente influenciada pelo conhecimento do fenômeno a ser mapeado, assim como das características da paisagem (fatores). Esse conhecimento determina a robustez da solução, para o processo de tomada de decisão, visto que influencia diretamente no nível de compensação necessário aos fatores.

2. O território do Município de Juquiá está, de maneira geral, sob elevado risco de deslizamento de terra, sendo necessária a constante atualização do mapeamento, com atenção especial às áreas classificadas com níveis de suscetibilidade alto e muito alto. $\mathrm{O}$

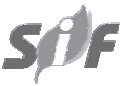

Revista Árvore, Viçosa-MG, v.38, n.6, p.973-982, 2014 
mapeamento fornece subsídios para o gerenciamento estratégico do Município de Juquiá, a fim de evitar tragédias humanas e perdas econômicas relacionadas ao fenômeno mapeado.

\section{REFERÊNCIAS}

ALHEIROS, M. M. et al. Manual de ocupação dos morros da região metropolitana do Recife. Recife: FIDEM, 2003. 384p.

AYALEW, L.; YAMAGISHI, H.; MARUI, H.; KANNO, T. Landslides in Sado Island of Japan: Part II. GIS-based susceptibility mapping with comparisons of results from two methods and verifications. Engineering Geology, v.81, p.432-445, 2005.

BERTONI, J.; LOMBARDI NETO, F.

Conservação do solo. 6.ed. São Paulo: Ícone, 2008. 355p.

BOROUSHAKI, S.; MALCZEWSKI, J. Implementing an extension of the analytical hierarchy process using ordered weighted averaging operator with fuzzy. Computer and Geosciences, v.34, n.4, p.399-410, 2008.

BOROUSHAKI, S.; MALCZEWSKI, J. Using the fuzzy majority approach for GIS-based multicriteria group decision-making. Computers \& Geosciences, v.36, n.3, p.302-312, 2010.

CARPI JUNIOR, S. Processos erosivos, recursos hídricos e riscos ambientais na bacia do rio Mogi-guaçu. 2001. 171f. Tese (Doutorado em Geociências e Meio Ambiente) - Instituto de Geociências e Ciências Exatas, Rio Claro, 2001.

CATANI, F.; CASAGLI, N.; ERMINI, L.; RIGHINI, G.; MENDUNI, G. Landslide hazard and riskmapping at catchment scale in the Arno River basin. Landslides, v.2, n.4, p.329-342, 2005.

CERVI, F.; BERTI, M.; BORGATTI, L.; RONCHETTI, F.; MANENTI, F.; CORSINI, A. Comparing predictive capability of statistical and deterministic methods for landslide susceptibility mapping: a case study in the northern Apennines (Reggio Emilia Province, Italy). Landslides, v.7, n.4, p.433-444, 2010.
DAS, S.; STEIN, A.; KERLE, N.; DADHWAL, V.K. Probabilistic landslide hazard assessment using homogeneous susceptible units (HSU) along a national highway corridor in the northern Himalayas, India. Landslides, v.8, n.3, p.293308, 2011.

DAS, I.; SAHOO, S.; VAN WESTEN, C.; STEIN, A.; HACK, R. Landslide susceptibility assessment using logistic regression and its comparison with a rockmass classification system, along a road section in the northern Himalayas (India),

Geomorphology, v.114, n.4, p.627-637, 2010.

EASTMAN, J. R. Decision strategy analysis. In: Idrisi kilimanjaro 2: Guide to GIS and image processing. Worcester: Clark University, 2003. v.2. p.1-22.

FEIZIZADEH, B.; BLASCHKE, T. Land suitability analysis for Tabriz County, Iran: a multi-criteria evaluation approach using GIS. Journal of Environmental Planning and Management, v.56, n.1, p.1-23, 2012.

FEIZIZADEH, B.; BLASCHKE, T. Uncertainty analysis of GIS-based ordered weighted averaging method for landslide susceptibility mapping in Urmia Lake Basin, Iran. Natural Hazard, v.65, n.3, p.2105-2128, 2013.

FEIZIZADEH, B.; BLASCHKE, T.; NAZMFAR, H.; MOGHADDAM, M. H. Landslide susceptibility mapping for the Urmia Lake Basin, Iran: A multicriteria evaluation approach using GIS.

International Journal of

Environmental Research, v.7, n.2, p.319336, 2013.

FERNANDES, N. F.; GUIMARÃES, R. F.; GOMES, R. A. T.; VIEIRA, B. C.; MONTGOMERY, D. R.; GREENBERG, H. Condicionantes geomorfológicos dos deslizamentos nas encostas: avaliação de metodologias e aplicação de modelo de previsão de áreas susceptíveis. Revista Brasileira de Geomorfologia, v.2, n.1, p.51-71, 2001.

FERNANDES, N. F.; GUIMARÃES, R. F.; GOMES, R. A. T.; VIEIRA, B. C.; MONTGOMERY, D. R.; GREENBERG, H. Topographic controls of landslides in Rio de Janeiro: Field evidence and modeling. Catena, v.55, n.2, p.163-181, 2004. 
GORSEVSKI, P. V; DONEVSKA, K. R, MITROVSKI, C. D. , FRIZADO, J. D. Integrating multi-criteria evaluation techniques with geographic information systems for landfill site selection: a case study using ordered weighted average. Waste Management, v.32, n.2, p.287-296, 2012.

INTARAWICHIAN, N.; DASANANDA, S. Analytical hierarchy process for landslide susceptibility mapping in lower Mae Chem watershed, Northern Thailand. Suranaree Journal Science Technologies, v.17, n.3, p.277-292, 2010.

LEI, Z.; JING-FENG, H. GIS-based logistic regression method for landslide susceptibility mapping in regional scale. Journal of Zhejiang University Science, v.7, n.2, p.2007-2017, 2006.

LEPSCH, I. F.; PRADO, H.; MENK, J. R. F.; SAKAI, E.; RIZZO, L. T. B. Levantamento de reconhecimento com detalhes dos solos da região do rio Ribeira de Iguape no estado de São Paulo. Campinas: IAC, 1999. mapa.

MALCZEWSKI, J., 1999. GIS and Multicriteria Decision Analysis. John Wiley, New York.

MALCZEWSKI, J. GIS-based land-use suitability analysis: a critical overview. Progress in Planning, v.62, n.1, p.3-65, 2004.

MALCZEWSKI, J. GIS-based multicriteria decision analysis: a survey of the literature. International Journal of Geographical Information Science, v.20, n.7, p.703-726, 2006.

MANTOVANI, F.; PASUTO, A.; SILVANO, S.; ZANNONI, A. Collecting data to define future hazard scenarios of the Tessina landslide.

International Journal of Applied

Observação da Terra e

Geoinformação, v.2, n.1, p.33-40, 2000.

MENG, Y.; MALCZEWSKI, J.; BOROUSHA, S. A GIS-based multicriteria decision analysis approach for mapping accessibility patterns of housing developmentsites: a case study in Canmore, Alberta. Journal of Geographic Information System, v.3, n.1, p.50-61, 2011.
RAUTELA, P.; LAKHERAZ, R. C. Landslide risk analysis between Giri and Tons Rivers in Himachal Himalaya (India). International Journal of Applied Observação da Terra e Geoinformação, v.2, n.3-4, p.153-160, 2000.

REMONDO, J.; BONACHEA, J.; CENDRERO, A. A statistical approach to landslide risk modelling at basin scale: from landslide susceptibility to quantitative risk assessment. Landslides, v.2, p.321-328, 2005.

SAATY, T. The analytic hierarchy process. New York: McGraw-Hill, 1980. 287p.

SCHOBBENHAUS, C. et al. Folhas SF23 Rio de Janeiro e SG23 Iguape. In: Carta Geológica do Brasil ao Milionésimo, Sistema de Informações Geográficas - SIG e 46 folhas na escala 1:1.000.000. Brasília: CPRM, 2004. CD ROM.

SILVEIRA, H. L. F.; VALENTE, R. O. A.; VETTORAZZI, C. A. Territorial planning of Juquiá municipality (state of São Paulo, Brazil), using an integrated analysis of the land cover and slope.. In: INTERNATIONAL CONFERENCE, 2008, Berlim. Book of abstracts. Berlim: Humbolt University, 2008. p.207.

SMYTH, C. G.; ROYLE, S. A. Urban landslide hazards: incidence and causative factors in Niterói, Rio de Janeiro State, Brazil. Applied

Geography, v.20, n.2, p.95-117, 2000.

SOARES, D. M. Geoprocessamento como ferramenta de análise do risco ao carreamento de sólidos para o rio Paraibuna em Juiz de Fora - MG. In: SIMPÓSIO BRASILEIRO DE SENSORIAMENTO REMOTO, 12., 2005, Goiânia. Anais... Goiânia: INPE, 2005. p.2375-2382.

TOMINAGA, L. K.; FERREIRA, C. J.; VEDOVELLO, R.; TAVARES, R.; SANTORO, J. Ocupação urbana e risco a processos de movimentos de massa no litoral norte de São Paulo: avaliação dos fatores geoambientais. In: SIMPÓSIO BRASILEIRO DE GEOGRAFIA FÍSICAAPLICADA, 11., 2005, São Paulo. Anais... São Paulo: Universidade de São Paulo, 2005. CD ROM.

USGS. National Landslide Information Center. Golden: Geologic Hazards Team, Apresentação, 2003.

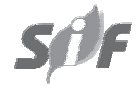


VALENTE, R. O. A.; VETTORAZZI, C. A.

Multicriteria evaluation in the definition of priority areas for forest restoration, aiming at the sustainable water management. In: BILIBIO, C.; HENSEL, O.; SELBACH, J. (Org.) Sustainable water management in the tropics and subtropics and case studies in Brazil. Jaguarão: Fundação Universidade Federal do Pampa, UNIKASSEL, PGCUlt, UFMA, 2011.v.1. p.377-406.

VALENTE, R. O.A.; VETTORAZZI, C.A. Definition of priority areas for forest conservation through the Ordered Weighted Averaging method, Forest Ecology and Management, v.256, p.14081417, 2008.

VALENTE, R. O. A.; VETTORAZZI, C. A. Avaliação multicriterial na definição de áreas prioritárias à restauração florestal, visando ao manejo sustentável da água. In: BRUN, A.L. et al. Sustentabilidade do uso da água nos trópicos e subtrópicos: estudos de casos brasileiros. Ijuí: UNIJUÍ, 2013. v.1. p.285244.

YALCIN, A. GIS-based landslide susceptibility mapping using analytical hierarchy process and bivariate statistics in Ardesen (Turkey): Comparisons of results and confirmations. Catena, v.72, n.1, p.1-12, 2008.

YAGER, R. R. On ordered weighted averaging aggregation operators in multicriteria decisionmaking. IEEE Systems, Man, and Cybernetics, v.18, n.1, p.183-190, 1988. 